Proceedings of SALT 23: 632-651, 2013

\title{
Children's knowledge of free choice inferences *
}

\author{
Peng Zhou \\ Macquarie University
}

\author{
Jacopo Romoli \\ Macquarie University
}

\author{
Stephen Crain \\ Macquarie University
}

\begin{abstract}
This paper presents experimental results showing that four-year-old Mandarinspeaking children draw free choice inferences from disjunctive statements, though they are not able to compute inferences of exclusivity for disjunctive statements or other scalar implicatures. The findings connect to those of Chemla \& Bott (2014) who report differences in how adults process free choice inferences versus scalar implicatures and, prima facie, the findings pose a challenge to treatments that attempt to unify inferences of both kinds. Instead, the findings appear to favour accounts that invoke different analyses for each kind of inference, such as Zimmerman 2000, Geurts 2005, and Barker 2010. The results, however, also support the recent approach in the experimental literature which attributes children's failures to compute scalar implicatures to a difficulty with alternatives: children may lack the lexical knowledge of alternatives, or these implicatures impose such a high processing cost that children are unable to handle the alternatives necessary to compute them (Gualmini, Crain, Meroni, Chierchia \& Guasti 2001; Chierchia, Crain, Guasti \& Thornton 2001; Reinhart 2006; Barner, Brooks \& Bale 2011; Singh, Wexler, Astle, Kamawar \& Fox 2012). If accessing alternatives is the source of children's difficulty, then they would be expected to perform better if the requisite alternatives are made explicit, as sub-strings of the asserted sentences. This is exactly what we found. Children were able to compute free choice inferences based on alternatives that were made explicit in the assertion, but children were unable to compute 'regular' scalar implicatures arising from alternatives lacking this property. We discuss the implications of these findings for the debate about the relationship between free choice inferences and scalar implicatures and children's knowledge of alternatives.
\end{abstract}

* For invaluable discussion and comments we thank Maria Aloni, Chris Barker, Cory Bill, Gennaro Chierchia, Emmanuel Chemla, Teresa Guasti, Drew Khlentzos, Manfred Krifka, Danny Fox, Clemens Mayr, Giorgio Magri, Enzo Moscati, Anna Notley, Louis Alonso-Ovalle, Francesca Panzeri, Hazel Pearson, Uli Sauerland, Greg Scontras, Raj Singh, Yasutada Sudo, Lyn Tieu, Rozz Thornton, the language acquisition group at Macquarie University, and the audiences at SALT 23, University of Milan-Bicocca, and University of Konstanz.

(C)2013 Zhou, Romoli, \& Crain 
Children's knowledge of free choice inferences

Keywords: Free choice, scalar implicatures, acquisition, alternatives

\section{Introduction}

A long standing puzzle in the semantics/pragmatics literature is how to account for the fact that disjunctive statements like (1a) license conjunctive inferences like (1b). ${ }^{1}$

a. Jack may have sushi or pasta.

b. $\quad \rightsquigarrow$ Jack may have sushi and Jack may have pasta

The fact that we infer (1b) from (1a) is surprising, because in modal logic a sentence of the form $\diamond(p \vee q)$ does not entail the corresponding sentence $\diamond p \wedge \diamond q$. Moreover, a plain disjunctive sentence like ( $2 \mathrm{a}$ ) never conveys the corresponding conjunctive inference in (2b); in fact, it typically conveys its negation, as we will see.

$$
\begin{aligned}
& \text { a. Jack had sushi or pasta. } \\
& \text { b. } \Varangle \rightarrow \text { Jack had sushi and pasta }
\end{aligned}
$$

The question, then, is why the addition of an existential modal to a disjunctive statement suddenly engenders inferences to the corresponding conjunctive sentence, as illustrated in (1). Inferences like (1b) are traditionally called 'free choice inferences,' the intuition being that sentence (2a) grants Jack free choice between the two options.

The derivation of free choice inferences has resulted in two main camps in the literature. We are interested in adjudicating between the predictions that each camp makes about the relationship between free choice inferences and scalar implicatures. Essentially, the difference between the two camps boils down to whether or not free choice inferences are equated with scalar implicatures. In particular, we have been investigating the predictions of those who would provide a unified analysis of free choice inferences and scalar implicatures. By grouping these phenomena together, the predictions of this camp are that, everything else being equal, we should expect uniform behavioral findings for both linguistic phenomena. Similar findings are expected in the acquisition of inferences of both kinds, as well as in adult language processing when adults are engaged in computing these inferences.

Chemla \& Bott (2014) have assessed this uniformity prediction in studies of language processing with adults, where scalar implicatures and free choice inferences are expected to manifest similar processing profiles. Taken at face value, however, their results fail to support the uniformity account. Different performance profiles were observed as adults computed free choice inferences as compared to scalar

1 See Kamp 1973; Zimmerman 2000; Geurts 2005; Fox 2007; Barker 2010; Klinedinst 2007; Chemla 2010; Chierchia 2013 among many others. 
implicatures. Following the example of Chemla \& Bott (2014), we have explored the uniformity account in language acquisition. The uniformity account predicts that, everything else being equal, children should show similar acquisition profiles in the development of the ability to compute free choice inferences and scalar implicatures. In line with the processing result by Chemla \& Bott (2014), however, the findings from our experimental investigations also fail to meet the expectations of the uniformity account. At this point, we have two options. First, we could simply conclude that the uniformity account is wrong. Alternatively, we could explore a response to the challenge that is posed by the experimental findings from studies of adults and children. In the second part of the paper, we pursue the second option. There we formulate a hypothesis about the observed behavioral differences. Our hypothesis is based on children's knowledge of alternatives, following suggestions in the literature, put forward by Chemla \& Bott (2014) and by others (Gualmini et al. 2001; Chierchia et al. 2001; Singh et al. 2012; Reinhart 2006; Barner et al. 2011). We then sketch the predictions that this hypothesis makes, and how we are proceeding to test these predictions.

The remainder of this introduction is organized as follows. We briefly introduce free choice inferences and scalar implicatures, and the debate that has ensued about their relationship. We also explain the different predictions for acquisition that follow from the different sides of the debate, and we sketch, as an example, one variant of the uniformity approach that attempts to unify scalar implicatures and free choice inferences, namely the account offered in Fox 2007. Section 2 describes the experiment, and section 3 discusses the implication of the results and formulates a possible response from the perspective of the uniformity account, based on alternatives. Section 4 concludes the paper.

\subsection{What are free choice inferences and scalar implicatures?}

Consider sentences (3a) and (3b). Example (3a) contains a plain disjunction, and example ( $3 b)$ contains an existential modal. When we hear these sentences we typically draw the corresponding inferences in (4a) and (4b), respectively.

a. Jack had sushi or pasta.

b. Jack may have sushi.

a. Jack didn't have both sushi and pasta.

b. Jack doesn't have to have sushi.

We will call the inference in (4b) the 'not-required-to' inference, and we will call the inference in (4a) the 'exclusivity' inference. Next consider sentence (5), which combines an existential modal and a disjunction. 
Children's knowledge of free choice inferences

Jack may have sushi or pasta.

Notice that an exclusivity inference is generally derived from (5), so the inference is that Jack may not have both sushi and pasta. Moreover, we also typically generate the conjunctive inference in (6).

(6) Jack may have sushi and Jack may have pasta.

This conjunctive inference is surprising because, as we have seen, from a plain disjunction like (3a), we never conclude the corresponding conjunction in (7). In fact, we typically conclude the negation of (7), namely (8). Clearly, the existential modal is responsible, at least in part, for the conjunctive inference from (5) to (6).

(7) Jack had sushi and Jack had pasta.

(8) Jack did not have both sushi and pasta.

The puzzle is to understand why the conjunctive inference arises when we combine an existential modal with disjunction. As mentioned earlier, the inference from (5) to (6) is traditionally called a 'free choice' inference, the intuition being that Jack has been given a free choice between sushi and pasta. Despite the name, this phenomenon extends beyond sentences with deontic modals, which grant permission. For example, such inferences extend to sentences such as the example in (9). Here the sentence expresses epistemic modality. ${ }^{2}$

(9) a. Jack might be in Sydney or in Wollongong.

b. $\quad \rightsquigarrow$ Jack might be in Sydney and might be in Wollongong

One characteristic of all three of the inferences under consideration is that they are suspendable. For instance, it is clear from the continuation in (10) that Jack doesn't have to have sushi. Similarly, we can suspend the exclusivity inference of the first statement in (11), by adding a continuation that makes it clear that it is possible that Jack had both sushi and pasta.

(10) Jack may have sushi. In fact, he might even have to have it.

2 Free choice inferences also arise with existential quantifiers and some generic sentences (see Fox 2007; Klinedinst 2007 and Nickel 2011 for discussion).

i. Some students take three semesters to complete it or do not finish it at all. (Fox 2007) $\rightsquigarrow$ Some students take three semesters and some students do not finish it at all

ii. Elephants live in Asia or in Africa.

(Nickel 2011)

$\rightsquigarrow$ Elephants live in Asia and elephants live in Africa 
(11) Jack had sushi or pasta. In fact, he might even have had both.

Finally, we can also suspend the free choice inference in the original example in (1a), repeated here as (12). As it is clear from the continuation, we are not saying that Jack has free choice between sushi and pasta; in view of the continuation, there is only one thing Jack can have, we just don't remember which one it is. ${ }^{3}$

$$
\text { Jack may have sushi or pasta. I don't remember which. }
$$

In the present paper we focus on the inferences in (13b) and (14b), which are generally regarded as being scalar implicatures, and free choice inferences like that in (15b). The question of interest is the relationship between free choice inferences and scalar implicatures. We turn to this question in the next section.

$$
\begin{aligned}
& \text { a. Jack may have sushi. } \\
& \text { b. } \text { Jack doesn't have to have it } \\
& \text { a. Jack had sushi or pasta. } \\
& \text { b. } ~ \rightsquigarrow \text { Jack didn't have both } \\
& \text { a. Jack may have sushi or pasta. } \\
& \text { b. } \quad \text { Jack may have sushi and may have pasta }
\end{aligned}
$$

\subsection{The debate \& predictions for acquisition}

So the question is: What is the relationship between free choice inferences and scalar implicatures? Two main answers have been offered in the literature. One approach contends that there is no relation between the two - they are completely different types of inferences. We will call this the 'ambiguity account,' because it poses some form of ambiguity in the interpretation of disjunction. Essentially, what this approach argues is that there is a reading of (16a) that simply entails (16b). In other words, free choice inferences are entailments of certain interpretations of sentences like (16a).

\section{(16) a. Jack may have sushi or pasta.}

3 Of course, not every inference is suspendable in this way. For instance, a conjunctive sentence like (iii) gives rise to the inference in (iv). But one cannot go on and suspend it as in (v).

iii. Jack had sushi and pasta.

iv. Jack had sushi.

v. But in fact it might be that he didn't have sushi. 
Children's knowledge of free choice inferences

b. Jack may have sushi and Jack may have pasta.

The second approach argues instead that there is an identity relation between free choice inferences and scalar implicatures - the two inferences are, in fact, the very same thing. We call this the 'scalar implicature account.' On this account, (16b) arises from (16a) as a scalar implicature. We will look a bit more at the details of these accounts, focusing especially on the scalar implicature (uniformity) account.

But first, let's spell out the predictions the alternative accounts make for the acquisition of free choice inferences and scalar implicatures. The ambiguity approach argues that free choice inferences and scalar implicatures are two completely different things. Therefore, it makes no clear predictions for acquisition, in the sense that any two different things could follow different acquisition paths, or they could accidentally overlap.

The scalar implicature approach, on the other hand, contends that free choice inferences are scalar implicatures. Therefore, everything else being equal, this approach makes a strong prediction, as formulated in (17).

Uniformity prediction for acquisition: children should behave uniformly with respect to free choice inferences and scalar implicatures.

We designed an experiment to test the prediction in (17). Before we turn to the experiment, it will be instructive to briefly sketch how one can derive free choice inferences as scalar implicatures.

\subsection{A scalar implicature-based approach to free choice}

This section offers an example of how free choice inferences can be derived as scalar implicatures. For the purposes of the example, we will adopt the theory proposed in Fox 2007, which argues that free choice inferences are a recursive type of scalar implicature. It should be understood that there are other scalar implicaturebased accounts, and some of them very different from Fox's theory, including accounts presented in Klinedinst 2007; Chemla 2010; van Rooij 2010; Franke 2011; Alonso Ovalle 2005; Chierchia 2013. Moreover, rather than going into Fox's theory in detail, we will simply sketch the intuition that underlies the theory.

It will be useful to begin with the idealized Gricean algorithm for computing scalar implicatures, as represented in (18). ${ }^{4}$ As (18) indicates, the basic idea is

4 Fox (2007) does not invoke this kind of pragmatically-based algorithm. In fact, based on observations about the generation of free choice inferences, he argues for an architecture of the semantics and pragmatics of scalar implicatures according to which scalar implicatures arise from the compositional side of meaning and not from the kind of pragmatic reasoning sketched in this section. This part of the theory is not relevant for our purposes, however, so we will ignore it here. In appendix A, we 
that when we hear an utterance, we reason about what the speaker might have said instead (among a restricted set of competitors). We then conclude that some of these competitors are false. More precisely, the competitors that are rendered false are ones that are not already entailed by the speaker's utterance.
a. The speaker said A.
b. The speaker might have said B.
c. It's false that B.

For instance, when we hear a disjunctive statement such as (19a), we reason that the speaker might have uttered the corresponding conjunctive statement (19b) instead, which would have been stronger, so we conclude that (19b) is false.

\section{a. Jack had sushi or pasta.}

b. Jack had sushi and pasta.

How do we proceed from here to computing free choice inferences as scalar implicatures? According to Fox (2007), there are two further ingredients. First, we need to consider more competitors for disjunctions (see Sauerland 2004). Second, the algorithm requires recursivity (see also Kratzer \& Shimoyama 2002; Alonso Ovalle 2005 among others).

The first ingredient is the consideration of more competitors. Take the sentence Jack had sushi or pasta in (19a). Not only is this compared to the alternative Jack had sushi and pasta in (19b), but it is also compared to the disjuncts it contains: Jack had sushi in (20a) and Jack had pasta in (20b).

$$
\begin{aligned}
& \text { a. Jack had sushi. } \\
& \text { b. Jack had pasta. }
\end{aligned}
$$

Although these alternatives are stronger that the assertion, if the algorithm in (18) is applied to these alternatives, the result would be the negation of the statements in (20), namely the assertion that Jack didn't have sushi and Jack didn't have pasta. Taken together, these negative statements would contradict the original assertion, Jack had sushi or pasta. Assuming that the speaker believes what she said, we would be led to conclude that the speaker is irrational, something we generally avoid. ${ }^{5}$ Therefore, nothing happens to the alternatives in (20), on the present account. However, in other cases these additional alternatives become crucial, as we will see.

In addition to a broader range of competitors, the second ingredient of the new

provide a more accurate reconstruction of Fox's (2007) account.

5 Notice that it does not follow from the simple algorithm that we have sketched above why we do not negate only one of the two disjuncts. Minimally, we need to add the condition that we cannot conclude that a competitor is false if this leads to the conclusion that another competitor is true. 
algorithm is a recursive step. The algorithm then assumes the following shape. As before, when we hear an utterance, we reason about what the speaker might have said (considering a restricted set of competitors). So far, everything is as it was, i.e., as in the algorithm in (18). However, according to the new algorithm, we also reason that, if the speaker had said one of the competitors, that competitor would have come with it own inferences. So, in making comparisons between the assertion and its competitors, not only are the plain competitor considered, but the comparisons are extended to include ones that assess the assertion against each competitor enriched with its inferences. The conclusion we draw is that the competitors, along with their associated inferences, are false. The algorithm is represented schematically in (21).

a. The speaker said A.

b. The speaker might have said B.

c. If the speaker had said B, B would have had C as an inference.

d. It's false that B and C.

To see how the algorithm works in practice, let us return to the example sentence (22a), Jack may have sushi or pasta. According to the new algorithm, the target assertion is compared to each of the disjuncts that are contained in the assertion, (22b) and (22c). As we saw, if these disjuncts were compared with the assertion using the older algorithm (18), they were negated on the threat of contradiction.
a. Jack may have sushi or pasta.
b. Jack may have sushi.
c. Jack may have pasta.

However, using the new algorithm, these competitor are compared with the assertion only after they have been enriched with their associated inferences. What inferences are associated with (22a) and (22c)? If the question under discussion is what Jack can have, and if sushi and pasta are relevant to the discussion, then from competitor (23a), Jack may have sushi, we would conclude (23b), Jack may not have pasta. Similarly, from (24a) we would conclude (24b), Jack may not have sushi.

a. Jack may have sushi.

b. Jack may not have pasta.

a. Jack may have pasta.

b. Jack may not have sushi.

In (25), we have combined the competitors and their inferences. These are among the comparison set for the example disjunctive sentence under consideration Jack may have sushi or pasta. 
a. Jack may have sushi but not pasta.

b. Jack may have pasta but not sushi.

Notice that both of the competitors (25a) and (25b) are stronger than the original assertion Jack may have sushi or pasta, so these wind up being negated by our algorithm. Therefore, we infer (26a) and (26b).

a. It's false that Jack may have sushi but not pasta.

b. It's false that Jack may have pasta but not sushi.

This is the last piece of the puzzle. From the assertion Jack may have sushi or pasta, the new algorithm allows us to infer (27).

Jack may have sushi and Jack may have pasta.

Following the new algorithm, we are therefore able to derive free choice inferences as a type of recursive scalar implicature. This concludes a brief sketch of one way that linguists have attempted to unify free choice inferences and scalar implicatures, based on Fox 2007. If this unification is on the right track, then the empirical prediction is that, everything else being equal, children should behave uniformly with respect to both linguistic phenomena. We turn now to the experiment that we conducted to test this prediction.

\section{The experiment}

\subsection{Methods}

\subsubsection{Participants}

Twenty-two Mandarin-speaking children (mean age 4;3, range 3;7-4;9) participated in our experiment.

\subsubsection{Procedure}

We used a Truth Value Judgement task (Crain \& Thornton 1998). In this task, one experimenter acts out a story using toy characters and props and a second experimenter plays the role of a puppet who says something about what happened in each story. The child participant's task is to say whether the puppet was right or wrong. 


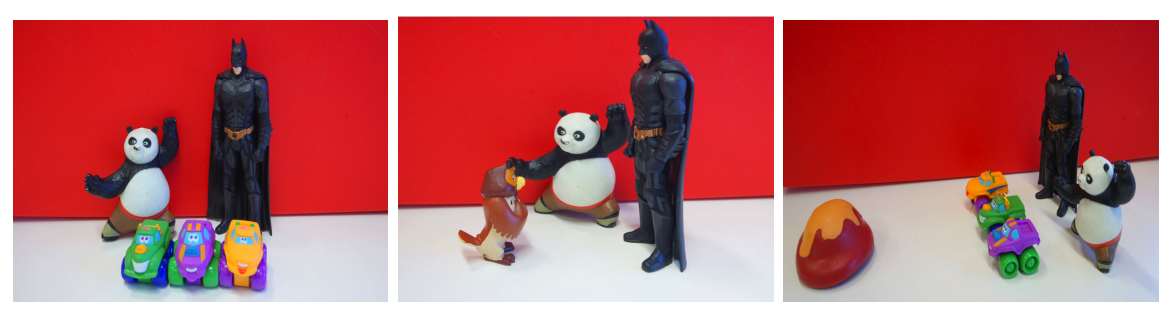

Figure 1 A free choice trial.

\subsubsection{Material}

The design of the experiment included two conditions, a free choice condition and a scalar implicature condition. Eight sentences were presented, four per condition. On a typical trial, Kung Fu Panda and Batman engaged in a racing competition (see Figure 1). Before the competition, Mr. Owl (the judge) explained the rules, telling Kung Fu Panda that he was only allowed to push the green car, and telling Batman that he was only allowed to push the orange car. But Kung Fu Panda and Batman were forgetful, so when the game was about to start, they asked the puppet to remind them of the rules. The puppet then produced the test sentence, as in (28).

Gongfu xiongmao keyi tui lüse xiaoche huozhe juse xiaoche.

Kung.Fu Panda may push green car or orange car

'Kung Fu Panda may push the green car or the orange car.'

The logic of the design is as follows: the relevant rule stated by Mr. Owl was (29), rendered schematically in (30).

(29) Kung Fu Panda may push the green car but may not push the orange one.

$$
\diamond p \wedge \neg \vee q
$$

The puppet's statement, on the other hand, was (28). On its literal meaning, (28) can be rendered simply as in (31), which is compatible with the rule in (30) (in fact it is entailed by (30)). However, we have seen that from the disjunctive statement (31) we can compute the free choice inference in (32), and this is not compatible with Mr. Owl's ruling, as rendered in (30).

$$
\begin{aligned}
& \diamond(p \vee q) \\
& \diamond p \wedge \diamond q
\end{aligned}
$$

Therefore, if the child participants compute free choice inferences of the form in (32), they are predicted to reject the puppet's statement. On the other hand, if 
children do not compute free choice inferences, they are expected to accept the puppet's disjunctive statement in (28) as an accurate description of Mr. Owl's ruling.

The very same logic applied in the scalar implicature condition. This condition came in two types. One testing the 'not-required-to' inference and the second testing the exclusivity inference. On two of the scalar implicature trials, the sentences contained the Mandarin modal verb keyi 'may' as in (33). In the context associated with the test sentence (33), Mr. Owl states that Winnie the Pooh must eat a green pepper. In other words, the context validates an assertion of the form $\square p$. The puppet's statement, on the other hand, is (33), which is of the form $\diamond p$. Again Mr. Owl's rule is compatible with the literal meaning of (33) but it is not compatible with its 'not-required-to' inference, $\neg \square p$. Therefore, once again, if the child participants compute the 'not-required-to' implicature, they are expected to reject the puppet's statement, but if child participants compute the literal meaning, but not the implicature, then they are expected to accept the puppet's statement.

$$
\begin{aligned}
& \text { Weinixiong keyi chi qingjiao. } \\
& \text { Winnie.the.Pooh may eat green pepper } \\
& \text { 'Winnie the Pooh may eat a green pepper'. }
\end{aligned}
$$

On the other type of control trials, the sentences contained the Mandarin disjunction word huozhe 'or' as in (34). On one typical trial, a red mermaid found both a red shell and a blue shell, which validates a statement of the form $p \wedge q$. The puppet's statement, however, was (34). Again the literal meaning $p \vee q$ is compatible with the events that unfolded in the story, but its exclusivity inference, $\neg(p \wedge q)$, is not. So, again, if children compute the exclusivity inference, they were expected to reject the puppet's statement, but not if they computed only the literal meaning.

Hongse meirenyu zhaodao-le baise beike huozhe lanse beike. red mermaid find-ASP white shell or blue shell 'The red mermaid found a white shell or a blue shell'.

\subsection{Results}

The results are reported in Figure 2. As this figure indicates, children rejected the puppet's statement in the free choice condition $91 \%$ of the time (on 80 out of 88 trials). By contrast, the same children rejected the test sentences in the scalar implicature condition only $18 \%$ of the time (on 16 out of 88 trials). A Wilcoxon Signed Ranks Test showed that there was a significant difference between conditions $(\mathrm{z}=4.52, \mathrm{p}<.001)$. 
Children's knowledge of free choice inferences

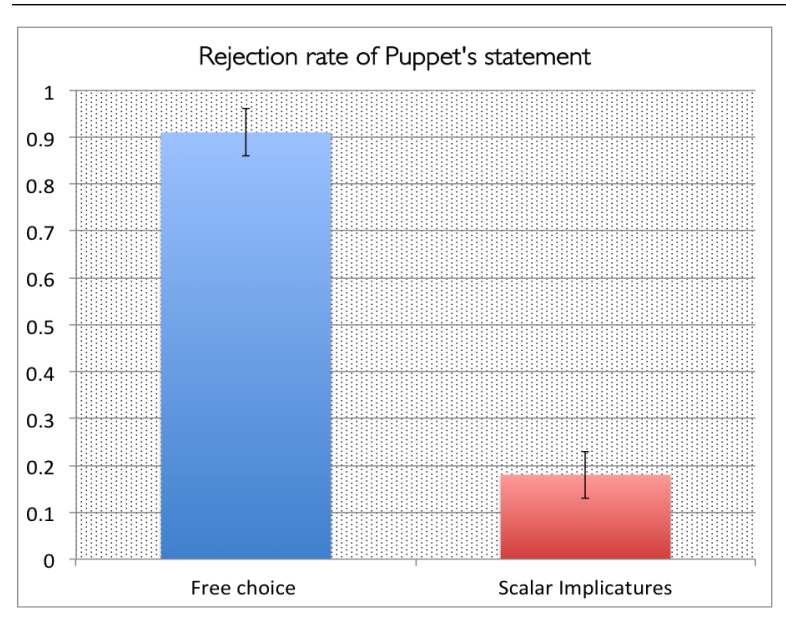

Figure 2 Rejection rate of the puppet's statement in the two conditions.

\section{General Discussion}

In the present experiment, children were found to compute free choice inferences, but not scalar implicatures. The scalar implicature result is consistent with previous findings in the literature (Gualmini et al. 2001; Chierchia et al. 2001; Papafragou \& Musolino 2003 among many others). However, the fact that children consistently computed free choice inferences is a novel finding. Moreover, the comparison between the two is the most informative part of the result, because this finding bears on the debate surrounding the relationship between free choice inferences and scalar implicatures.

The findings reveal that children's behaviour with free choice inferences and scalar implicatures is not uniform. We have, therefore, two options at this point. First, we can conclude that the ambiguity camp was right and that free choice inferences and scalar implicatures are simply two different things. Second, we could maintain the conclusion of the uniformity account - that free choice inferences are scalar implicatures - but in this case we are required to invoke some other factor in order to explain children's different behaviour in response to these linguistic phenomena. While the first strategy is the most straightforward way of accounting for our results, we think that there are compelling arguments previously raised in the literature for the scalar implicature-based account (see Chemla \& Bott 2014 for discussion).

Therefore, in the following, we have chosen to explore a response to the present findings based on the uniformity account. In particular, we formulate an hypothesis based on a suggestion by Chemla \& Bott (2014) and elsewhere in the literature, namely that children have access to a restricted set of alternatives (Singh et al. 2012; 
Chemla \& Bott 2014; Reinhart 2006; Barner et al. 2011).

\subsection{The restricted alternatives hypothesis}

In this section, we explore one kind of response that can be made to the data that challenge the scalar implicature-based approach. We call this hypothesis the 'restricted alternatives hypothesis.' The hypothesis has two main ingredients. One is the observation that the alternatives from which scalar implicatures and free choice inferences are derived are different. The second is the observation that explicitly mentioning alternatives helps children to successfully compute their corresponding inferences. Let us start with the first observation - that the alternatives from which we derived the 'not-required-to' inference are different than the alternatives used to compute scalar implicatures. The alternatives from which we derived the notrequired-to and the scalar implicatures in (35c) and (36c) from (35a) and (36a) were (35b) and (36b), respectively. To obtain these alternatives from the corresponding assertions, we need to replace the modal or the connective.

a. Jack may have sushi.

b. Jack has to have sushi.

c. $\rightsquigarrow$ Jack doesn't have to have sushi

a. Jack had sushi or pasta.

b. Jack had sushi and pasta.

c. $\rightsquigarrow$ Jack didn't have sushi and pasta

For the free choice inference, on the other hand, we only needed the alternatives corresponding to the disjuncts. ${ }^{6}$ In other words, the alternatives that we need for free choice inferences are substrings of the assertion, while those of other scalar implicatures are not.
a. Jack may have sushi or pasta.
b. Jack may have sushi.
c. Jack may have pasta.
d. $\rightsquigarrow$ Jack may have sushi and Jack may have pasta

The second ingredient of the restricted alternatives hypothesis is the observation in the literature that mentioning alternatives helps children compute their corresponding inferences (Gualmini et al. 2001; Chierchia et al. 2001; Reinhart 2006). For instance, in Gualmini et al. 2001, children were presented with disjunctive sentences when their corresponding conjunctions were true. In other words, when the context

6 This is true in particular in the theory by Fox (2007), but most scalar implicature-based accounts have this same property. 
validated that Jack had sushi and pasta, the puppet would say (38) and, as previously shown, children, unlike adults tend to accept this sentence in this context.

$$
\text { Jack had sushi or pasta. }
$$

This has been taken as evidence that they do not compute scalar implicatures from (38). On the other hand, if in the same context children are given both (38) and (39) by different puppets, they prefer (39). And this can be interpreted, following Gualmini et al. 2001, as evidence that when children are explicitly provided with the alternatives, they are able compute their corresponding inferences.

$$
\text { Jack had sushi and pasta. }
$$

Finally, let us add the simple observation that if alternatives are contained in the assertion they are necessarily 'mentioned' in the assertion, so to speak. Now, putting together the two ingredients, we can formulate the hypothesis as in (40) (see Gualmini et al. 2001; Chierchia et al. 2001; Reinhart 2006; Singh et al. 2012; Barner et al. 2011; Chemla \& Bott 2014).

Restricted alternatives hypothesis: Children compute inferences arising from alternatives which are substrings of the assertion.

Schematically here is how the restricted alternatives hypothesis in (40) can account for the findings of the present experiment. The alternatives of free choice inferences are mentioned in the assertion, in the sense just explained, while those of other scalar implicatures are not. Mentioning the alternatives helps children compute their corresponding inferences. Therefore, we expect a difference in children's behaviour with free choice inferences versus scalar implicatures. In sum, the restricted alternatives hypothesis can account for our results, without abandoning the uniformity account.

Of course, just formulating the hypothesis in (40) is not enough. We need to test its predictions further. There are two predictions in particulars that we are exploring. The first one is the prediction that other inferences arising from alternatives mentioned in the assertion should be computed by children. The second is the prediction that if we can find free choice inferences that do not arise from alternatives that are mentioned in the assertion, then children should not compute them (in the same way as they do not compute exclusivity and 'not-required-to' implicatures).

\section{Conclusion}

We presented experimental findings revealing that four-year-old children can compute free choice inferences, but not scalar implicatures. This is challenging for 
the approach that argues that they are the same thing. We explored a response to this challenge that we called the restricted alternatives hypothesis. This is based on two ingredients: (a) the alternatives from which free choice inferences arise are mentioned in the assertion but those of scalar implicatures are not (b) mentioning alternatives help children compute their corresponding inferences. There are two immediate predictions of this hypothesis that need to be tested further. One prediction concerns other inferences that arise from alternatives mentioned in the assertion - these are predicted to be successfully computed by children. Second, free choice inferences that do arise from alternatives that are not explicitly mentioned in the assertion should, however, not be computed by children. In conclusion, the present study is an example of how experimental work on acquisition can constrain theoretical debate. The experiment was designed to address a theoretical approach that groups free choice inferences and scalar implicatures together. The finding represented a challenge for this account, because children's behaviour was not uniform across the two kinds of inferences. We have tried to respond to this challenge, in order to maintain the uniformity account, at least until follow-up experimental studies are conducted.

\section{Appendix A: free choice as recursive scalar implicatures}

In this section, we provide the computation of free choice inferences as recursive scalar implicatures (as in Fox 2007) in more detail and following more closely the original implementation. Fox (2007) argues for an exhaustification-based approach to scalar implicatures (see also Chierchia, Fox \& Spector 2012; Magri 2011 among many others). In his version of this approach, scalar implicatures are obtained through an exhaustivity operator, indicated as EXH. EXH applies to a proposition and its associated alternatives. It affirms the proposition while negating a subset of the alternatives, the 'excludable' ones.

$$
\left[\left[\operatorname{EXH}_{\mathscr{A} l t}(p)\right]\right](p)=\lambda w \cdot p(w) \wedge \forall q \in \mathscr{E} x c l(p, \mathscr{A} l t(p))[\neg q(w)]
$$

The excludable alternatives $\mathscr{E} x c l(p, \mathscr{A} l t(p))$ are defined as in (42): all the alternatives that are not entailed by the assertion and the exclusion of which does not lead to the inclusion of another alternative.

$$
\mathscr{E} x c l(p, \mathscr{A} l t(p))=\{q \in \mathscr{A} l t(p): p \nsubseteq q \wedge \neg \exists r[r \in \mathscr{A} l t(p) \wedge(p \wedge \neg q) \subseteq r]\}
$$

We can now go back to (43a) and see how we can derive (43b) via a recursive application of the exhaustivity operator above.

a. Jack may have sushi or pasta.

b. Jack may have sushi and may have pasta. 
Children's knowledge of free choice inferences

Consider the schematic version of (43a) in (44a) and its alternatives in (44b).

$$
\begin{aligned}
& \text { a. } \diamond(p \vee q) \\
& \text { b. } \mathscr{A} t_{1}(\diamond(p \vee q))=\left\{\begin{array}{l}
\diamond[p \vee q] \\
\diamond p \\
\diamond q \\
\diamond[p \wedge q]
\end{array}\right\}
\end{aligned}
$$

The result of exhaustifying (44a) with respect to (44b) is (45).

$$
\operatorname{EXH}[\diamond(p \vee q)]=\diamond(p \vee q) \wedge \neg \diamond(p \wedge q)
$$

Consider now what happens if we exhaustify again: the alternatives that we have to consider are the exhaustifications of the alternatives in $\mathscr{A} l t_{1}$, represented in (46). Notice in particular the exhaustification of $\nabla p$ and $\nabla q$, which is carried out with respect to the alternatives in $\mathscr{A} l t_{1}$. Crucially, in the case of $\diamond p$, we negate $\diamond q$ and vice versa.

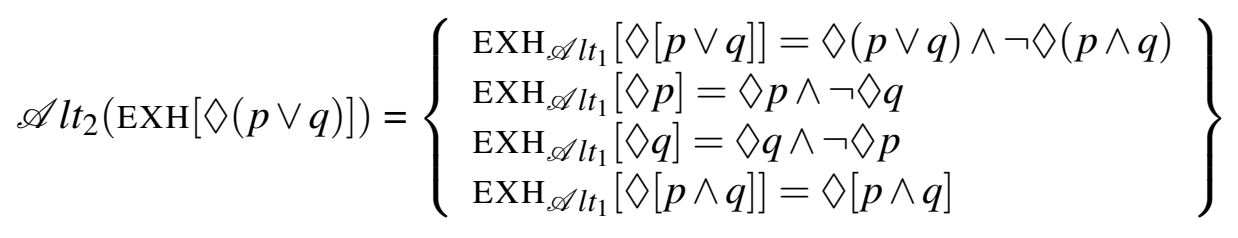

At this point, we now exhaustify again with respect to this second set of alternatives and in this way we obtain the free choice inference $\diamond p \wedge \diamond q$. This is because when we negate the exhaustification of $\diamond p$ and $\diamond q$, we obtain a biconditional that, together with the assertion, entails the conjunction of $\diamond p$ and $\diamond q$.

$$
\begin{aligned}
& \mathrm{EXH}_{\mathscr{A} l t_{2}}\left[\mathrm{EXH}_{\mathscr{A} l t_{1}} \diamond[p \vee q]\right]= \\
& {[\diamond(p \vee q) \wedge \neg \diamond(p \wedge q)] \wedge \neg(\diamond p \wedge \neg \vee q) \wedge \neg(\diamond q \wedge \neg \diamond p)=} \\
& {[\diamond(p \vee q) \wedge \neg \diamond(p \wedge q)] \wedge(\diamond p \rightarrow \diamond q) \wedge(\diamond q \rightarrow \diamond p)=} \\
& {[\diamond(p \vee q) \wedge \neg \diamond(p \wedge q)] \wedge(\diamond p \leftrightarrow \diamond q)=} \\
& \diamond p \wedge \diamond q
\end{aligned}
$$

\section{Appendix B: two versions of the restricted alternatives hypothesis}

Above we discussed the hypothesis in (48) based on the idea that children have a restricted access to alternatives.

Restricted alternatives hypothesis: Children compute inferences arising from alternatives which are substrings of the assertion. 
There are at least two elements of variation in this hypothesis. First, there is the question of why children have restricted access to alternatives. The options proposed in the literature range from lack of lexical knowledge or processing limitation problems to a combination of the two (see Barner et al. 2011 for discussion). Second, there is a question as to what it is that children do with the restricted set of alternatives they have access to. One answer to this question is that they simply do not compute scalar implicatures, as they are lacking the crucial alternatives that would give rise to them. A different answer is to say that they do compute scalar implicatures, but these are simply different from those of adults. This second version is defended and tested further in Singh et al. 2012. In both cases, the idea is that for something of the form in (49) children can only access the alternatives in (50) while adults have the set in (51).

$$
p \vee q
$$

$$
\begin{aligned}
& \text { children's alternative: }\{p \vee q, p, q\} \\
& \text { adults' alternatives: }\{p \vee q, p, q, p \wedge q\}
\end{aligned}
$$

The observation by Singh et al. (2012) is that if we apply the recursive exhaustification mechanism by Fox (2007) (see Appendix A) to (49) and the children's alternatives in (50a) we obtain the conjunctive inference in (53). On the other hand, if we do it to (49) and the adults' alternatives in (51), we obtain the regular exclusivity inference in (53). To illustrate, consider what happens when we exhaustify $p \vee q$ with respect the alternatives $\{p \vee q, p, q\}$. The result of one round of exhaustification is vacuous as indicated in (52).

$$
\mathrm{EXH}[p \vee q]=p \vee q
$$

Consider now what happens if we exhaustify again: the alternatives that we have to consider are the exhaustifications of the alternatives in (51), represented in (53).

$$
\mathscr{A} l t_{2}(\operatorname{EXH}[(p \vee q)])=\left\{\begin{array}{l}
\operatorname{EXH}_{\mathscr{A}} l t_{1}[p \vee q]=(p \vee q) \\
\operatorname{EXH}_{\mathscr{A}} l t_{1}[p]=p \wedge \neg q \\
\operatorname{EXH}_{\mathscr{A}} l t_{1}[q]=q \wedge \neg p
\end{array}\right\}
$$

In parallel to the derivation of free choice in Appendix A, when we exhaustify with respect to this second set of alternatives we obtain the conjunctive inference $p \wedge q$. This is because when we negate the exhaustification of $p$ and $q$, we obtain a biconditional that, together with the assertion, entails the conjunction of $p$ and $q$.

$$
\begin{aligned}
& \operatorname{EXH}_{\mathscr{A} l t_{2}}\left[\mathrm{EXH}_{\mathscr{A} l t_{1}}[p \vee q]\right]=(p \vee q) \wedge \neg(p \wedge \neg q) \wedge \neg(q \wedge \neg p)= \\
& (p \vee q) \wedge(p \rightarrow q) \wedge(q \rightarrow p)=
\end{aligned}
$$


Children's knowledge of free choice inferences

$$
\begin{aligned}
& (p \vee q) \wedge(p \leftrightarrow q)= \\
& p \wedge q
\end{aligned}
$$

The idea, therefore, is that children accept a sentence like (49) in a situation in which both $p$ and $q$ are true not (only) because they do not compute the exclusivity inference in (56), but because they compute the conjunctive inference in (55).

$$
\begin{aligned}
& p \wedge q \\
& \neg(p \wedge q)
\end{aligned}
$$

Our results are compatible with both versions of the restricted alternatives hypothesis. Moreover, they support the assumption by Singh et al. (2012) that children can compute recursive exhaustification. We leave for further research experimentally testing the two versions of the restricted alternatives hypothesis.

\section{References}

Alonso Ovalle, Luis. 2005. Disjunction in alternative semantics. UMass PhD dissertation.

Barker, Chris. 2010. Free choice permission as resource-sensitive reasoning. Semantics and Pragmatics 3(10). 1-38.

Barner, David, Neon Brooks \& Alan Bale. 2011. Accessing the unsaid: the role of scalar alternatives in children's pragmatic inferences. Cognition 188. 87-96.

Chemla, Emmanuel. 2010. Similarity: towards a unified account of scalar implicatures, free choice permission and presupposition projection. Unpublished manuscript.

Chemla, Emmanuel \& Lewis Bott. 2014. Processing inferences at the semantics/pragmatics frontier: disjunctions and free choice. Cognition 130(3). 380396.

Chierchia, Gennaro. 2013. Logic in Grammar: Polarity, Free Choice, and Intervention. Oxford University Press.

Chierchia, Gennaro, Stephen Crain, Maria Teresa Guasti \& Rosalind Thornton. 2001. 'Some' and 'or:' a study on the emergence of logical form. In Sarah Catherine Howell, Sarah Fish \& Thea Keith-Lucas (eds.), Boston University Conference on Language Development (BUCLD) 24, 22-44. Somerville, Massachusetts: Cascadilla Press.

Chierchia, Gennaro, Danny Fox \& Benjamin Spector. 2012. The grammatical view of scalar implicatures and the relationship between semantics and pragmatics. In Claudia Maienborn, Klaus von Heusinger \& Paul Portner (eds.), Semantics: An International Handbook of Natural Language Meaning, 2297-2332. Berlin: Mouton de Gruyter. 
Crain, Stephen \& Rosalind Thornton. 1998. Investigations in Universal Grammar: A Guide to Experiments on the Acquisition of Syntax and Semantics. Cambridge, Massachusetts: The MIT Press.

Fox, Danny. 2007. Free choice and the theory of scalar implicatures. In Uli Sauerland \& Penka Stateva (eds.), Presupposition and Implicature in Compositional Semantics, 71-120. Palgrave.

Franke, Michael. 2011. Quantity implicatures, exhaustive interpretation, and rational conversation. Semantics and Pragmatics 4(1). 1-82. doi:10.3765/sp.4.1.

Geurts, Bart. 2005. Entertaining alternatives: Disjunctions as modals. Natural Language Semantics 13(4). 383-410.

Gualmini, Andrea, Stephen Crain, Luisa Meroni, Gennaro Chierchia \& Maria Teresa Guasti. 2001. At the semantics/pragmatics interface in child language. In Rachel Hastings, Brendan Jackson \& Zsofia Zvolenszky (eds.), Semantic and Linguistic Theory (SALT) 21, 231-247. Cornell University, Ithaca, NY: CLC Publications.

Kamp, Hans. 1973. Free choice permission, vol. 74 57-74. Aristotelian Society, Methuen.

Klinedinst, Nathan. 2007. Plurality and possibility. UCLA PhD dissertation.

Kratzer, Angelika \& Junko Shimoyama. 2002. Indeterminate pronouns: The view from Japanese. In Yukio Otsu (ed.), Tokyo Conference on Psycholinguistics, vol. 3, 1-25. Tokyo: Hituzi Syobo.

Magri, Giorgio. 2011. Another argument for embedded scalar implicatures based on oddness in DE environments. Semantics and Pragmatics 4(6). 1-51.

Nickel, Bernhard. 2011. Generically free choice. Linguistics and Philosophy 33(6). 479-512.

Papafragou, Anna \& Julien Musolino. 2003. Scalar implicatures: experiments at the semantics-pragmatics interface. Cognition 86. 253-282.

Reinhart, Tanya. 2006. Interface Strategies. Optimal and Costly Derivations. Cambridge, Mass.: MIT Press.

van Rooij, Robert. 2010. Conjunctive interpretation of disjunction. Semantics and Pragmatics 3(11). 1-28. doi:10.3765/sp.3.11.

Sauerland, Uli. 2004. Scalar implicatures in complex sentences. Linguistics and Philosophy 27(3). 367-391.

Singh, Raj, Ken Wexler, Andrea Astle, Deepthi Kamawar \& Danny Fox. 2012. Children interpret disjunction as conjunction. consequences for the theory of scalar implicatures. Under review.

Zimmerman, Thomas Ede. 2000. Free choice disjunction and epistemic possibility. Natural Language Semantics 8(255-290). 
Children's knowledge of free choice inferences

Peng Zhou

Department of Cognitive Science

Australian Hearing Hub

16 University Avenue

Macquarie University NSW 2109

peng.zhou@mq.edu.au

Stephen Crain

Department of Cognitive Science

Australian Hearing Hub

16 University Avenue

Macquarie University NSW 2109

stephen.crain@mq.edu.au
Jacopo Romoli

Department of Cognitive Science Australian Hearing Hub

16 University Avenue

Macquarie University NSW 2109

jacopo.romoli@mq.edu.au 